\title{
ANTIQUARK POLARIZATION INSIDE THE PROTON IS SMALL*
}

\author{
T. P. Cheng ${ }^{\dagger}$ and Ling-Fong $\mathrm{Li}^{\diamond}$ \\ ${ }^{\diamond}$ Department of Physics, Carnegie Mellon University, Pittsburgh, \\ $P A 15213$ \\ ${ }^{\dagger}$ Department of Physics and Astronomy, University of \\ Missouri, St Louis, MO 63121
}

\begin{abstract}
Quark contributions to the proton spin as deduced from polarized DIS of leptons off a nucleon target, and the octet baryon magnetic moments, can be used to deduce the antiquark polarizations $\Delta_{\bar{q}}$ inside the proton. In this way the 1992 analysis by Karl is shown to imply $\Delta_{\bar{q}} \simeq 0$. Such a spin structure fits nicely into the chiral quark interpretation of the proton spin and flavor puzzles.
\end{abstract}

\section{Introduction}

In the last several years, starting with EMC目 in the late 1980's, the polarized deep inelastic lepton-nucleon scattering experiments have revealed a nucleon spin structure that deviates significantly from the simple quark model expectation. It is particularly puzzling in view of the fact that the same spin structure in the nonrelativistic constituent quark model seems to lead to a reasonably good description of baryon magnetic moments 2 . In this paper we re-examine the proton spin data in connection with the octet baryon magnetic moments. We find that such an investigation can lead to some useful insight into the proton spin structure, above and beyond what the (spin-dependent structure function) $g_{1}$ sum ruld and the baryonic weak axial vector couplings can tell us. Namely, this combined analysis

\footnotetext{
* CMU-HEP95-15, DOE-ER/40682-104, hep-ph/9510\#\#\#
} 
suggests that the antiquarks inside the proton are not significantly polarized. And, as we shall show, this lends further support to the chiral quark model 5 approach to the various nucleon structure puzzles 6 , ?

In Sec.2, we first discuss the simple model where the magnetic moment of a baryon comes from those of its constituent quarks. The flavor- $S U(3)$ is used to express the octet baryon moments in terms of the intrinsic $u, d$, and $s$ magnetic moments and the quark and antiquark contributions to the proton spin. In Sec.3, the "proton spin crisis" is briefly recalled. We then show, in Sec.4, that an analysis made by Karl 8 actually contains definite information about the antiquark polarizations in the proton. In Sec.5 the spin and magnetic moment structure is studied in the chiral quark model. Finally we conclude and discuss the implication of our result.

\section{Spin structure and magnetic moments}

Let us first discuss the relation between the quark and antiquark polarization contributions to the proton spin and the proton magnetic moment. One usually denotes the $q$-flavor contribution to the proton spin as:

$$
\Delta q=\left(q_{\uparrow}-q_{\downarrow}\right)+\left(\bar{q}_{\uparrow}-\bar{q}_{\downarrow}\right) \equiv \Delta_{q}+\Delta_{\bar{q}}
$$

where $q_{\uparrow}$ is the number of $q$-flavor quarks with spin parallel, and $q_{\downarrow}$ anti-parallel, to the proton spin. Thus $\Delta_{q}$ and $\Delta_{\bar{q}}$ are the quark and antiquark polarizations, respectively.

For the $q$-flavor quark contribution to the proton magnetic moment, we have however

$$
\mu_{p}(q)=\Delta_{q} \mu_{q}+\Delta_{\bar{q}} \mu_{\bar{q}}=\left(\Delta_{q}-\Delta_{\bar{q}}\right) \mu_{q} \equiv \widetilde{\Delta_{q}} \mu_{q}
$$

where $\mu_{q}$ is the magnetic moment of the $q$-flavor quark. The negative sign simply reflects the opposite quark and antiquark moments, $\mu_{\bar{q}}=-\mu_{q}$, as in general $\mu_{q}=e_{q} g_{q} / 2 m_{q}$, and when we go from quark to antiquark only the charge $e_{q}$ changes sign, but not the mass $m_{q}$ or the gyromagnetic ratio $g_{q}$. Thus the spin factor that enters into the expression for the magnetic 
moment is $\widetilde{\Delta q}$, the difference of the quark and antiquark polarizations. If we assume that the proton magnetic moment is entirely built up from the light quarks inside it, we have

$$
\mu_{p}=\widetilde{\Delta u} \mu_{u}+\widetilde{\Delta d} \mu_{d}+\widetilde{\Delta s} \mu_{s}
$$

In such an expression there is a separation of the intrinsic quark magnetic moments and the spin wavefunctions. Flavor- $S U(3)$ symmetry then implies, the proton wavefunction being related the $\Sigma^{+}$wavefunction by the interchange of $d \leftrightarrow s$ and $\bar{d} \leftrightarrow \bar{s}$ quarks, the relations $(\widetilde{\Delta u})_{\Sigma^{+}}=(\widetilde{\Delta u})_{p} \equiv \widetilde{\Delta u},(\widetilde{\Delta d})_{\Sigma^{+}}=\widetilde{\Delta s}$, and $(\widetilde{\Delta s})_{\Sigma^{+}}=\widetilde{\Delta d}$; similarly it being related to the $\Xi^{0}$ wavefunction by a further interchange of $u \leftrightarrow s$ quarks, thus $(\widetilde{\Delta d})_{\Xi^{0}}=(\widetilde{\Delta d})_{\Sigma^{+}}=$ $\widetilde{\Delta s},(\widetilde{\Delta s})_{\Xi^{0}}=(\widetilde{\Delta u})_{\Sigma^{+}}=\widetilde{\Delta u}$, and $(\widetilde{\Delta u})_{\Xi^{0}}=(\widetilde{\Delta s})_{\Sigma^{+}}=\widetilde{\Delta d}$. We have,

$$
\begin{aligned}
& \mu_{\Sigma^{+}}=\widetilde{\Delta u} \mu_{u}+\widetilde{\Delta s} \mu_{d}+\widetilde{\Delta d} \mu_{s}, \\
& \mu_{\Xi^{0}}=\widetilde{\Delta d} \mu_{u}+\widetilde{\Delta s} \mu_{d}+\widetilde{\Delta u} \mu_{s},
\end{aligned}
$$

the intrinsic moments $\mu_{q}^{\prime} s$ being unchanged when we go from Eq.(3) to Eqs.(4) and (5). The $n, \Sigma^{-}$, and $\Xi^{-}$moments can be obtained from their isospin conjugate partners $p, \Sigma^{+}$, and $\Xi^{0}$ by the interchange of their respective $u \leftrightarrow d$ quarks: $(\widetilde{\Delta u})_{\Sigma^{-}}=(\widetilde{\Delta d})_{\Sigma^{+}}=\widetilde{\Delta s}$, etc.

$$
\begin{aligned}
\mu_{n} & =\widetilde{\Delta d} \mu_{u}+\widetilde{\Delta u} \mu_{d}+\widetilde{\Delta s} \mu_{s}, \\
\mu_{\Sigma^{-}} & =\widetilde{\Delta s} \mu_{u}+\widetilde{\Delta u} \mu_{d}+\widetilde{\Delta d} \mu_{s}, \\
\mu_{\Xi^{-}} & =\widetilde{\Delta s} \mu_{u}+\widetilde{\Delta d} \mu_{d}+\widetilde{\Delta u} \mu_{s} .
\end{aligned}
$$

The relations for the $I_{z}=0, Y=0$ moments are more complicated in appearance but the underlying arguments are the same.

$$
\begin{aligned}
& \mu_{\Lambda}=\frac{1}{6}(\widetilde{\Delta u}+4 \widetilde{\Delta d}+\widetilde{\Delta s})\left(\mu_{u}+\mu_{d}\right)+\frac{1}{6}(4 \widetilde{\Delta u}-2 \widetilde{\Delta d}+4 \widetilde{\Delta s}) \mu_{s} \\
& \mu_{\Lambda \Sigma}=\frac{-1}{2 \sqrt{3}}(\widetilde{\Delta u}-2 \widetilde{\Delta d}+\widetilde{\Delta s})\left(\mu_{u}-\mu_{d}\right) .
\end{aligned}
$$

The proton Eq.(3) and neutron Eq.(6) were first written down by Sehgal 1 , and its generalization to other octet baryons have been discussed by a number of authors 10 . Since we will be 
following most closely Karl's work 8 , we shall refer to Eqs.(3) to (10) as the Karl-Sehgal (KS) equations. We should however note that the equations actually written down by Karl involve the polarization sum $\Delta q=\Delta_{q}+\Delta_{\bar{q}}$ rather than the difference $\widetilde{\Delta q}=\Delta_{q}-\Delta_{\bar{q}}$ because he has chosen to work with an "effective quark magnetic moment" $\mu_{q}^{\prime}=\widetilde{\Delta q} \mu_{q} / \Delta q$. The resultant equation $\mu_{p}(q)=\Delta q \mu_{q}^{\prime}$, instead of Eq.(2), thus does not separate the spin-wavefunction and the quark intrinsic moments. Namely, the $\mu_{q}^{\prime}$ moments, just as $\Delta q$ and $\widetilde{\Delta q}$, depend on the spin wavefunction as well as the intrinsic moments. Thus different approaches have been adopted in Ref. 8 and the present paper. We are of the opinion that, in the effort to express all the baryon moments in terms of the proton spin factors, our assumption of a complete separation of the spin wavefunctions and the intrinsic quark moments is the more reasonable approach.

\section{Simple quark model results and the measured proton spin structure}

In the nonrelativistic constituent quark model, there is no quark sea. The proton spin follows simply from the addition of its valence quark spins. One finds:

$$
\Delta u=\frac{4}{3}, \quad \Delta d=-\frac{1}{3}, \quad \Delta s=0, \quad \Delta \Sigma=1
$$

where $\Delta \Sigma=\Delta u+\Delta d+\Delta s$ is the total quark contribution to the proton spin. There is no strange quark inside proton, hence no strange quark polarization, $\Delta s=0$. Also, there is no antiquark, hence no antiquark polarization, $\Delta_{\bar{q}}=0$. This means that in the simple quark

model (sQM) we have $\Delta q=\widetilde{\Delta q}$. The proton and neutron magnetic moments, for example, are given by Eqs.(3), (6), and (11), as

$$
\begin{aligned}
& \mu_{p}=\frac{4}{3} \mu_{u}-\frac{1}{3} \mu_{d}=\frac{m_{N}}{m_{u, d}} g \mu_{N} \\
& \mu_{n}=-\frac{1}{3} \mu_{u}+\frac{4}{3} \mu_{d}=-\frac{2}{3} \frac{m_{N}}{m_{u, d}} g \mu_{N}
\end{aligned}
$$

where we have assumed the equality of $u$ and $d$ constituent quark masses, denoted by $m_{u, d}$, as well as their gyromagnetic ratios $g_{u}=g_{d}=g$ (for example, $g=2$ for Dirac particles). $\mu_{N}$ 
is the nucleon magneton, and $m_{N}$ the nucleon mass. From this we deduce the famous $S U(6)$ result 11 for the moment ratio of $\mu_{p} / \mu_{n}=-1.5$, which is to be compared to the experimental value of $\left(\mu_{p} / \mu_{n}\right)_{\text {exptl }}=-1.48$. Furthermore with a measured value of $\mu_{p}=2.79 \mu_{N}$, Eq.(12) suggests that $m_{u, d}$ is about a third of the nucleon mass. In this way the simple constituent quark model actually provides a reasonable fit to all the octet moments (Table 1), especially if we allow for a heavier strange constituent quark mass, $m_{u, d} / m_{s} \simeq 0.6$.

However, in 1988, EMC reported their measurement of the proton structure function $g_{1}(x)$, which seemed to suggest a quark contribution to the proton spin very different from this simple quark model expectation (11). Using $S U(3)$ symmetry and baryonic weak axialvector couplings $g_{A}=1.254, F / D=0.632$ and the $g_{1}$ sum rule, one could deduce the various quark contributions to the proton spin:

$$
\Delta u=0.75 \pm 0.12, \quad \Delta d=-0.51 \pm 0.12, \quad \Delta s=-0.22 \pm 0.12, \quad \Delta \Sigma=0.02 \pm 0.21 .
$$

The discrepancy between the phenomenological values (14) and the quark model expectations (11) - especially the indication of the presence in the proton a strongly polarized strange quark sea, and the possibility that proton gets almost none of its spin from quarks — caused it to be called the "proton spin crisis". It is certainly puzzling why the simple quark model spin structure (11) can lead to a satisfactory description of the baryon magnetic moments, and yet fails to be in agreement with a more direct measurement (14)?

\section{A combined analysis of the proton spin and baryon magnetic moment data}

Instead of viewing this discrepancy as a definitive failure of the constituent quark model, we consider it as showing that for certain phenomena the effects of the quark sea can be very important. The issue is then what are the properties of the quark sea do the observations imply, and what mechanism can produce such a quark sea. We may start with the following questions: 
(i) Does a good fit to the baryon magnetic moments necessarily mean that the quark contributions to the proton spin must have the sQM values (11)? Or, does the measured spin factors (14) yield a good (or even better) fit to the magnetic moments?

(ii) As both spin factors $\Delta q^{\prime} s$ and magnetic moments $\mu_{B}^{\prime} s$ are related to the quark polarizations inside the proton, can an analysis using $(\Delta q)_{\text {exptl }}$ and $\mu_{B}$ values lead to further insights into the proton spin structure, above and beyond what each set can reveal?

It turned out that the analysis performed in 1992 by Karl 8 had gone a long way in answering these questions. What Karl did was to use the KS equations: (3) to (10), to search for the values of $(\widetilde{\Delta q})^{\prime} s$ so that the best-fit (in the sense of lowest $\chi^{2}$, etc.) to all the measured baryon magnetic moments could be obtained. He found the following set:

$$
\widetilde{\Delta u}=0.86 \pm 0.12, \quad \widetilde{\Delta d}=-0.40 \pm 0.12, \quad \widetilde{\Delta s}=-0.20 \pm 0.12, \quad \widetilde{\Delta \Sigma}=0.27 \pm 0.21
$$

The fit it produces is better than the simple quark model fit. See Table 1 for more detail. Thus the answer to Question-(i) is that the required magnetic moment best-fit values (15) are actually closer to the measured spin values (14) than $(\Delta q)_{\mathrm{sQM}}$ of (11).

In the meantime, the EMC result has been extended by further analysist, by new measurements by SMC, E142 and E143려, extending to neutron target, to larger kinematic regime, with better statistics. With even higher order QCD correction 13 included, we now know that Bjorken sum ruld 3 is verified 4 to an accuracy about $12 \%$, and, although the original EMC result (14) has been confirmed in general terms, with improved accuracy the values of the proton spin components have been slightly modified. In particular, the total quark contribution to the proton spin is no longer being consistent with zero. The more recent result14 is

$$
\Delta u=0.83 \pm 0.06, \quad \Delta d=-0.42 \pm 0.06, \quad \Delta s=-0.10 \pm 0.06, \quad \Delta \Sigma=0.31 \pm 0.11
$$

which is even more similar-in-value to the $\widetilde{\Delta q} s$ of Eq.(15) than the values known in 1992 when Karl performed his best-fit analysis. This closeness-in-value between Eq.(16)'s $(\Delta q)_{\text {exptl }}$ and Eq.(15)'s $(\widetilde{\Delta q})_{\mu_{B}}$ immediately allows us to infer that antiquark polarization 
inside the proton $\Delta_{\bar{q}}=\left(\bar{q}_{\uparrow}-\bar{q}_{\downarrow}\right)$ is small. In more quantitative terms, the antiquark to quark polarization fraction $\delta_{\bar{q}}$ can be expressed as the difference and sum ratio of the two kinds of spin factors, $\Delta q$ and $\widetilde{\Delta q}$. From Eqs.(1) and (2), we have

$$
\delta_{\bar{q}}=\frac{\Delta_{\bar{q}}}{\Delta_{q}}=\left|\frac{\Delta q-\widetilde{\Delta q}}{\Delta q+\widetilde{\Delta q}}\right| .
$$

After substituting in the values (15) and (16), and combing errors by quadrature, we have

$$
\delta_{\bar{u}}=0.02 \pm 0.08, \quad \delta_{\bar{d}}=0.02 \pm 0.16, \quad \delta_{\bar{s}}=0.33 \pm 0.45
$$

They are all consistent with being zero, although the error on the anti-strange quark polarization is quite large. Still, this result is suggestive that the polarization of the antiquarks in the quark-sea is suppressed.

As explained at the end of Sec.2, Eqs.(3) to (10) as written down by Karl 1 have $\Delta q^{\prime} s$ in place of $\widetilde{\Delta q} s$. Thus he was not able to make the connection to the antiquark polarization result as discussed in this paper.

\section{Spin and magnetic moment in the chiral quark model}

The measured values $\Delta q^{\prime} s$ in Eq.(16) are all smaller than the sQM prediction (11). Thus the quark sea must have the following specific features: for each flavor, the quark sea must be strongly polarized in the direction opposite to the proton spin, and yet the antiquarks in this sea are not much polarized. We shall show that the chiral quark model produces just such a quark sea.

The basic idea of chiral quark model is that the energy scale associated with chiral symmetry breaking $\Lambda_{\chi S B} \simeq 1 \mathrm{GeV}$ is much larger than that associated with QCD confinement $\Lambda_{Q C D} \simeq 0.1-0.3 \mathrm{GeV}$. The distance scale in between these two nonperturbative QCD thresholds just corresponds to the interior of a hadron (but not so short a distance when perturbative QCD becomes operative). In this intermediate non-perturbative QCD regime, the relevant degrees of freedom are the quasiparticles of quarks, gluons, and the Goldstone 
bosons associated with the spontaneous breaking of the $S U(3) \times S U(3)$ chiral symmetry. Here, the quarks propagate in a ground state filled with the collective excitations of $q \bar{q}$ condensates and in this way gain a large constituent quark mass. The Goldstone bosons are the usual pseudoscalar mesons, but propagating here in the interior of the hadron. (we shall refer to them as the internal Goldstone bosons.) The quark-gluon interactions of the underlying QCD bring about chiral symmetry breaking and the Goldstone modes of excitations. However, when the description is reorganized in terms of the quasiparticle effective fields of constituent quarks and Goldstone bosons, we expect that the gluons will now have a negligibly small effective coupling. Thus, the only important interaction is the coupling among the Goldstone bosons and quarks.

A quark sea created through internal Goldstone boson (GB) emissions by a valence quark,

$$
q_{\uparrow} \rightarrow G B+q_{\downarrow}^{\prime} \rightarrow\left(q \overline{q^{\prime}}\right)_{0} q_{\downarrow}^{\prime}
$$

has just the desired spin polarization features. The coupling of the pseudoscalar Goldstone boson to the quarks will flip the polarization of the quark: $q_{\uparrow} \rightarrow q_{\downarrow}^{\prime}$. We note that the final state $q_{\downarrow}^{\prime}$ carries all the polarization of the produced quark-sea, as the pair $\left(q \overline{q^{\prime}}\right)_{0}$ - coming out of the Goldstone boson - must be in the spin-zero combination:

$$
\left(q \overline{q^{\prime}}\right)_{0}=\frac{1}{\sqrt{2}}\left(q_{\uparrow} \overline{q_{\downarrow}^{\prime}}-q_{\downarrow} \overline{q_{\uparrow}^{\prime}}\right) .
$$

The quark sea created by such a mechanism will be polarized, as given by $q_{\downarrow}^{\prime}$, in a direction opposite to the proton spin, and the produced antiquark $\overline{q^{\prime}}$ must be unpolarized (i.e. equal probability for $\overline{q_{\uparrow}^{\prime}}$ and $\overline{q_{\downarrow}^{\prime}}$ ) as shown in (20).

In a previous publication the have shown that the broken- $U(3)$ version of the chiral quark model with two parameters (the octet and singlet Goldstone bosons couplings to the quarks) can provide a simple and unified account of the proton's spin and flavor puzzles. The quark contributions to the proton spin have been calculated to be

$$
\Delta u=\frac{4}{3}-\frac{1}{9}\left(37+8 \varsigma^{2}\right) a, \quad \Delta d=-\frac{1}{3}-\frac{2}{9}\left(1-\varsigma^{2}\right) a, \quad \Delta s=-a .
$$


where $a \propto\left|g_{8}\right|^{2}$ is the probability for a $u$-quark to emit a $\pi^{+}$[and its $S U(3)$ generalizations], $\varsigma=g_{1} / g_{8}$ is the singlet and octet Goldstone boson coupling ratio. We have shown that with a choice, for example, of $a=0.1$ and $\varsigma=-1.2$, this model yields a $\bar{u}-\bar{d}$ asymmetry compatible with the observed violation of the Gottfried sum rule 15 , and the observed asymmetry in the proton-neutron Drell-Yan processes 16 , etc. Such parameters then yield, through Eq.(21) the spin values as

$$
\Delta u=0.79, \quad \Delta d=-0.32, \quad \Delta s=-0.10, \quad \Delta \Sigma=0.37
$$

to be compared to the phenomenological values (16).

Before discussing all the magnetic moments, let us first show that the well-known $S U(6)$ result for the proton-neutron ratio is maintained in the chiral quark model. Substituting (21) into Eqs.(3) and (6) we have

$$
\frac{\mu_{p}}{\mu_{n}}=\left(-\frac{3}{2}\right)\left[1-\frac{5}{6} a\left(1-\frac{m_{u, d}}{m_{s}}\right)\right]
$$

i.e. the $S U(6)$ result is preserved in the flavor- $S U(3)$ limit of $m_{u, d}=m_{s}$.

That our result (22) is reasonably close to the $(\Delta q)_{\text {exptl }}^{\prime}$ of (16), which are in turn close-

in-value to $(\widetilde{\Delta q})_{\mu_{B}}^{\prime}$ s of $([15)$, leads us to expect that this model should be able to give a satisfactory description of the baryon magnetic moments as well. In the last column of Table 1 the numerical values for the illustrative parameters of $a=0.10$ and $\varsigma=-1.2$ are presented. One should keep in mind that our's is an $S U(3)$ symmetric calculation, as it is based on KS equations and the $S U(3) \times U(1)$ symmetric chiral quark model. The only $S U(3)$ breaking effect that has been taken into account is the different constituent masses $m_{u, d} \neq m_{s}$ in the quark intrinsic moments. Thus we do not really expect a better than 20 to $30 \%$ agreements from the model predictions.

\section{Discussion}

The KS equations, whether used in the simple constituent quark model, the chiral quark model, or the best-fit program by Karl, give a reasonably good account of $\mu_{B}^{\prime} s$. We interpret 
this fact to mean that the basic idea of the baryon magnetic moment being built up from the constituent quark moments is a robust framework. We have thus neglected possible contribution to the baryon magnetic moment by the orbital motion of the quarks. The success of the fits may be viewed as an a posteriori justification of this assumption. In this framework, we have shown that the antiquarks inside the proton are not significantly polarized. And, such a spin structure fits nicely into the chiral quark model approach where the antiquarks are produced through the emissions of the spin-zero internal Goldstone bosons.

Many authors 17 have suggested that the deviation of the "observed" quark spin contribution $(\Delta q)_{\text {exptl }}$ from the simple quark model prediction $(\Delta q)_{\mathrm{sQM}}$ is due to a possible gluonic polarization $\Delta G$. These authors argued that one must first subtract out this gluonic term in order to reveal the "true" quark contributions, $(\Delta q)_{\text {exptl }}=(\Delta q)_{\text {true }}-\frac{\alpha_{s}}{2 \pi} \Delta G$, and they suggest that only then do we expect agreement with the quark model expectation, $(\Delta q)_{\text {true }}=(\Delta q)_{\mathrm{sQM}}$. According to this approach, one would then predict, because of the simple quark model property of $\Delta q=\widetilde{\Delta q}$, that the best-fit $(\widetilde{\Delta q})_{\mu_{B}}^{\prime}$ s should be close in value to the $(\Delta q)_{\mathrm{sQM}}^{\prime}$ of (11). Karl has shown that (11) actually is not as a good fit as the values of in (15). We interpret it to mean that this particular scenario with a significant $\Delta G$ is not favored by the magnetic moment data.

On the other hand, the broken- $U(3)$ version of the chiral quark model can account for all these spin and magnetic moment data, as well as the flavor structure, in a simple and natural way. This list of positive attributes of the chiral quark model has been enhanced by the independent suggestion that Goldstone boson exchanges can provide a better description of the fine-structure features of the hadron spectroscopy 18 . To us, all these results suggest that the original nonrelativistic quark model is basicly correct in its description of the low energy hadron physics. It only needs to be augmented by a light quark sea which is generated perturbatively by the valence quarks through internal Goldstone boson emissions. This approach to the fundamental problem of proton structure warrants further investigation.

This work is supported at CMU by the US Department of Energy (DE-FG02-91ER- 
40682), and at UM-St Louis by the National Science Foundation (PHY-9207026).

1. J. Ashman, et al.(EMC), Phys. Lett. B206 (1988) 364; Nucl. Phys. B328 (1990) 1.

2. See, for example, textbook reviews by D. H. Perkins, Introduction to High Energy Physics, 3rd ed., Addison-Wesley, Reading, MA (1992); H. Georgi, Weak Interactions and Modern Particle Theory, Benjamin/Cummings, Menlo Park, CA (1984).

3. J. D. Bjorken, Phys. Rev. 148 (1966) 1479.

4. J. Ellis and R. L. Jaffe, Phys. Rev. D 9 (1974) 1444.

5. A. Manohar and H. Georgi, Nucl. Phys. B234 (1984) 189.

6. E. J. Eichten, I. Hinchliffe, and C. Quigg, Phys. Rev. D 45 (1992) 2269.

7. T. P. Cheng and L. F. Li, Phys. Rev. Lett. 74 (1995) 2872.

8. G. Karl, Phys. Rev. D 45 (1992) 247.

9. L. M. Sehgal, Phys. Rev. D 10 (1974) 1663.

10. See, for example, J. Bartelski and R. Rodenberg, Phys. Rev. D 41 (1989) 2800, and other citations in Ref. 8.

11. M. A. B. Beg, B. W. Lee, and A. Pais, Phys. Rev. Lett. 13 (1964) 514.

12. B. Adeva et al.(SMC), Phys. Lett. B302 (1993) 533; P. L. Anthony et al. (E142), Phys. Rev. Lett. 71 (1993) 959; K. Abe et al. (E143), Phys. Rev. Lett. 74 (1995) 346.

13. J. Kodaira et al., Phys. Rev. D 20 (1979) 627; S. A. Larin et al., Phys. Rev. Lett. 66 (1991) 862; A. L. Kataev and V. V. Starshenko, Mod. Phys. Lett. A 10 (1995) 235. 
14. J. Ellis and M. Karliner, Phys. Lett. B341 (1995) 397.

15. P. Amaudruz et al.(NMC), Phys. Rev. Lett. 66 (1991) 2712.

16. A. Baldit et al(NA51), Phys. Lett. B332 (1994) 244.

17. G. Altarelli and G. Ross, Phys. Lett. B212 (1988) 391; see also, A. V. Efremov and O. V. Teryaev, Dubna Report No. E3-88-387 (1988); C. S. Lam and B. A. Li, Phys. Rev. D 25 (1982) 683.

18. L. Ya Glozman and D. O. Riska, hep-ph 9505422, submitted to Phys Report. 
TABLE 1 Different fits to the baryon magnetic moments via KS equations.

The simple quark model and Karl's best-fit results are from Ref. $\mathrm{B}$, with the quark model having a $\chi^{2} / D F=7.35 / 5$ compared to the best-fit with $4.42 / 4$. Here the $\mu_{q}^{\prime} s$ are among the parameters being varied to produce the fits. Thus no specific assumption about the gyromagnetic ratio such as $g_{q}=2$ has been made. The last column is meant to illustrate the ability of the chiral quark model to account for $\mu_{B}^{\prime}$ s. We have taken $\Delta q=\widetilde{\Delta q}$ with values as given in (22), and have, with the constraint of $\mu_{u}=-2 \mu_{d}$ and $\mu_{s} / \mu_{d}=0.6$, adjusted the remaining one independent value of the $\mu_{q}^{\prime} s$ to get a good fit.

\begin{tabular}{|c|ccc|c|}
\hline & magnetic & simple & Karl's & chiral quark model \\
& moments quark model best-fit & $a=0.1, \varsigma=-1.2$ \\
\hline \hline$p$ & 2.79 & 2.68 & 2.69 & 2.69 \\
$n$ & -1.91 & -1.92 & -1.85 & -1.88 \\
$\Sigma^{+}$ & 2.48 & 2.55 & 2.59 & 2.56 \\
$\Sigma^{-}$ & -1.16 & -1.13 & -1.22 & -1.10 \\
$\Xi^{0}$ & -1.25 & -1.40 & -1.33 & -1.37 \\
$\Xi^{-}$ & -0.68 & -0.48 & -0.61 & -0.48 \\
$\Lambda$ & -0.61 & -0.59 & -0.59 & -0.60 \\
$\Lambda \Sigma$ & -1.60 & -1.60 & -1.53 & -1.58 \\
\hline$\mu_{u}$ & & 1.76 & 2.42 & 2.74 \\
$\mu_{d}$ & & -1.00 & -1.21 & -1.37 \\
$\mu_{s}$ & & -0.61 & 0.71 & 0.82 \\
\hline
\end{tabular}

